\title{
Enhancement of Laminar Mixing in Stirred Vessel using Geometrical Perturbations
}

\author{
A. Hidalgo-Millán ${ }^{1}$, B. Taboada ${ }^{2}$, L. Vega-Alvarado ${ }^{3}$, R. Zenit ${ }^{4}$, Gabriel Ascanio ${ }^{* 3}$ \\ ${ }^{1}$ Unidad Académica de Ciencias Básicas e Ingeniería \\ Universidad Autónoma de Nayarit, Tepic, Nayarit, México \\ ${ }^{2}$ Instituto de Biotecnología \\ Universidad Nacional Autónoma de México, Cuernavaca, Morelos, México \\ ${ }^{3}$ Centro de Ciencias Aplicadas y Desarrollo Tecnológico \\ Universidad Nacional Autónoma de México \\ México, D. F., México \\ *gabriel.ascanio@ccadet.unam.mx \\ ${ }^{4}$ Instituto de Investigaciones en Materiales \\ Universidad Nacional Autónoma de México \\ México DF, México
}

\begin{abstract}
Geometrical perturbations by using single and coaxial impellers configurations have been investigated in the laminar regime $(R e=2.68)$ in unbaffled stirred vessels. Combinations of one and two coaxial radial discharge flow impellers under centered and eccentric conditions were studied with viscous Newtonian fluid. The study of geometrical perturbations has been made in terms of the mixing times, energy consumption and the flow fields. Homogenization degrees and mixing times, determined with a color-discoloration technique, were evaluated by means of image processing. The flow fields were investigated using the particle image velocimetry technique. The use of off-centered impellers increases the pumping capacity; however, little effect of eccentricity was obtained when using two coaxial impellers under off-centered conditions obtaining the best results with a novel configuration consisting of a dual turbine with connecting plates.
\end{abstract}

Keywords: Mixing, laminar flow, visualization, hydrodynamics, stirred vessels, eccentric impellers.

\section{RESUMEN}

Se presentan los resultados de la investigación en tanques agitados en régimen laminar $(R e=2.68)$ con impulsores sencillos y coaxiales. Para ello se han elegido combinaciones de uno y dos agitadores de descarga radial centrados y descentrados con respecto al tanque empleando fluidos newtonianos. El estudio se llevó a cabo en términos del tiempo de mezclado mediante la técnica de colorimetría y procesamiento de imágenes. Los campos de flujo se obtuvieron por medio de la técnica de velocimetría de imágenes de partículas (PIV por sus siglas en inglés). Se observó un incremento en la capacidad de bombeo empleando un impulsor excéntrico. Sin embargo, el efecto fue menor al emplear dos impulsores coaxiales en posición descentrada. Por tal motivo se propuso una nueva configuración a base de dos agitadores coaxiales conectados mediante un par de barras.

\section{Introduction}

Mixing in stirred vessels is a key unit operation found in many industrial processes such as chemical, pharmaceutical, paints, pulp and paper, etc. This operation is usually carried out under steady flow conditions with the impeller centered with respect to the vessel centerline and rotating steadily in one direction only. From a hydrodynamic standpoint, steady mixing at low Reynolds number is commonly inefficient due to the flow structures generated from the beginning of the process. The presence of isolated regions has been noticed when using open impellers operating under laminar regime [1]. In the case of fluids possessing yield stress or inelastic Newtonian fluids well-mixed regions surrounded by stagnant fluid, known as caverns or pseudo caverns, respectively, are generated around the impeller. On the other hand, quasi-static or dead zones are also formed far from the impeller. Several techniques have been developed and used to visualize flow patterns in stirred vessels and to reveal the existence, location and size of mixing malfunctions [2]. The simplest 
technique for the qualitative analysis of flow patterns and the measurement of mixing times is colorimetry, first reported by Norwood and Metzner [3]. This non-intrusive technique is based on an acid/base indicator reaction. From a quantitative standpoint, particle image velocimetry (PIV) is perhaps the most used technique for observing flow patterns. It basically consists of comparing two consecutive pictures of an illuminated plane taken at very short times. For that purpose, the flow is seeded with light-reflecting tracers, which are tracked by means of image processing software. Although PIV is a sophisticated visualization technique used with transparent fluids, it provides reliable information on the flow behavior. X-ray [4] or electrical resistance tomography [5] are good alternatives when using non-transparent fluids such as concentrated suspensions.

These mixing malfunctions are in some cases persistent and they remain visible even if the working fluid is stirred for long periods. The easiest way to achieve the degree of homogenization required for the process by minimizing the effect of the aforementioned flow structures consists of increasing the rotational speed combined with the use of baffled tanks, which leads to an increase of the power drawn by the impeller. For some applications, especially those in which the turbulent regime must not be achieved because of the air incorporation or the fluid is shear sensitive, an increase of the impeller speed may damage the material to be mixed, therefore different alternatives must be considered. It has been demonstrated that the fluid can be thoroughly homogenized if the flow is continuously perturbed [6]. Geometrical perturbations based on the use of off-centered impellers and dynamic perturbations by using time-dependent rotating impellers or impellers rotating alternatively in both directions have been used to mix fluids in shorter times [7]. Both geometrical and dynamic perturbations are based on the pioneering work of Aref [8], who demonstrated that the chaotic flow prevents the formation of coherent regions in the vicinity of offcentered cylinders. These results were later confirmed by Aref and Balachandar [9] and Franjione et al. [10]. Alvarez et al. [11] and Ascanio et al. [7] showed that segregated regions are readily destroyed even if the agitation shaft is slightly displaced from the vessel centerline.
Montante et al. [12] studied numerically and experimentally the effect of the impeller eccentricity on mixing performance in unbaffled vessels in the turbulent regime, finding that geometrical perturbations inducing complex features to flow patterns. Karcz et al. [13] showed that mixing times decrease and power consumption increases with the impeller offcentered. Recently, Galletti and Brunazzi [14] and Galletti et al. [15] studied the effect of shaft eccentricity in an unbaffled tank in the turbulent regime, confirming the presence of two vortical structures, one above and one below the impeller. Moreover, they found that flow instabilities have a significant impact on macro-mixing.

On the other hand, dynamic perturbations were first reported by Ottino et al. [16], Swanson and Ottino [17] and later by Muzzio et al. [18, 19]; they found that homogenization could be enhanced in off-centered cylinders rotating alternatively in the two directions during short times. These findings were later applied to stirred vessels by Ascanio et al. [7] and Ascanio and Tanguy [20]. They found that the operating conditions, namely the impeller speed and the rotation period play a fundamental role in the mixing performance with Newtonian and non-Newtonian fluids. Lamberto et al. [21] showed also that the continuous flow perturbation causes chaotic flow in stirred vessels minimizing the effect of the flow structures generated in the beginning resulting in a high efficient mixing process.

Chaotic flow caused by geometrical perturbations is indeed an attractive alternative for enhancing mixing in laminar stirred vessels. The aim of the present work is to investigate the performance of a mixing arrangement based on the use of geometrical perturbations inducing unsteady mixing compared with the traditional configuration with a centered impeller rotating steadily.

\section{Materials and methods}

\subsection{Mixing system}

Figure 1a shows the experimental setup used for this work. The mixing system consists basically of a transparent cylinder made of polycarbonate having an inner diameter $(T)$ of $165 \mathrm{~mm}$ and 210 $\mathrm{mm}$ height unbaffled. The liquid height is equal to 
the vessel diameter $(H=D=165 \mathrm{~mm})$. In order to avoid significant changes on the refraction index and reduce optical distortions, as well as maintain the fluid temperature, the vessel is surrounded by a square jacket containing the same fluid under study. Because the impeller rotates at very low speed, no increase of the working fluid temperature was noticed. A radial flow discharge impeller (Rushton turbine) having a diameter of 55 $\mathrm{mm}(1 / 3 T=D)$ was fitted to a right shaft driven by a $1 / 2 \mathrm{hp}$ DC motor, whose speed was carefully controlled in a close-loop mode. A torquemeter was placed between the motor and the agitation shaft, so that power drawn by the impeller was continuously registered.

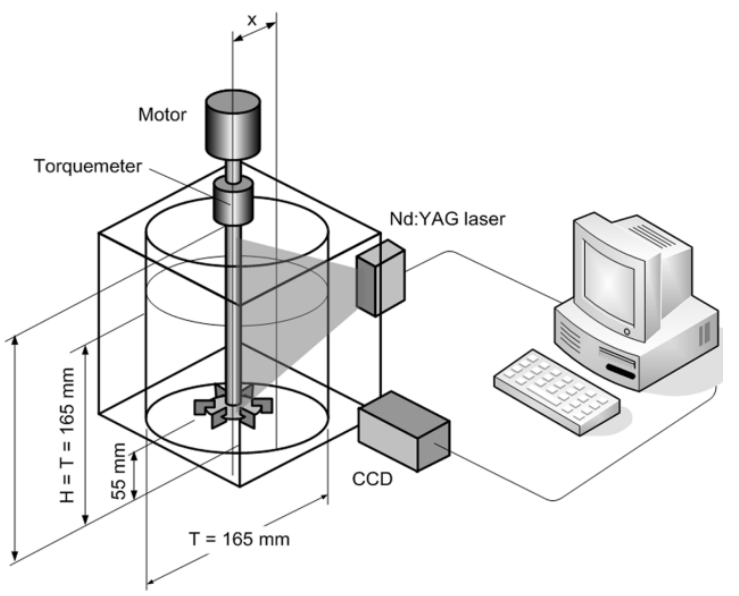

(a)
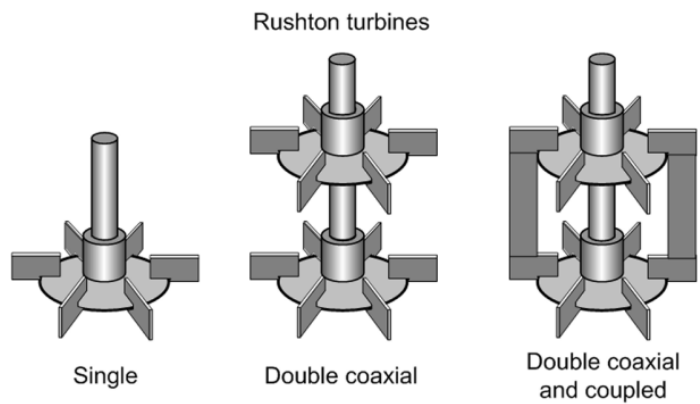

(b)

Figure 1. (a) Experimental setup PIV array; (b) configuration of impellers.

Three different impeller configurations were used for the tests, namely: a) single Rushton turbine; b) double coaxial Rushton turbines; and c) double coaxial Rushton turbines coupled by two thin plates spaced at $180^{\circ}$ (see Figure 1b). Each turbine consists of six square blades of $15.5 \mathrm{~mm}$ equally spaced around a disc. The clearance from the tank bottom and the distance between impellers was $1 / 3 T$. The tests were performed under steady and unsteady flow conditions by placing the impellers centered and off-centered. The off-centered condition defined by $X^{*}=x / r$, where $x$ is the distance from the vessel centerline to the impeller radial position $(x=25 \mathrm{~mm})$ and $r$ is the impeller radius $(D / 2)$, thus $X^{*}=0.3$. In all cases, the impeller rotated at a constant rotational speed $(\mathrm{N})$ of $1 \mathrm{rps}$ resulting in a tip speed of $0.17 \mathrm{~m} / \mathrm{s}$ and a Reynolds number $\left(\operatorname{Re}=\rho N D^{2} / \mu\right) \operatorname{Re}=2.68$.

\subsection{Fluids and mixing time measurement}

Pure glycerol (USP grade) having a density $(\rho)$ of $1250 \mathrm{~kg} / \mathrm{m}^{3}$ and a dynamic viscosity $(\mu)$ of 1.41 $\mathrm{Pa} \cdot \mathrm{s}$ was used as working fluid. Mixing times were evaluated by means of a color-discoloration nonintrusive technique based on a fast acid-base reaction, which has been described elsewhere [3, $7,21]$. Green bromocresol was used as tracer and small amounts of $4 \mathrm{M} \mathrm{HCl}$ or $4 \mathrm{M} \mathrm{NaOH}$ were added for changing the $\mathrm{pH}$ resulting in color change of the fluid contained in the stirred vessel. For the test performed in this work, blue and orange colors corresponded to acidic and basic media, respectively. Although small amounts of $\mathrm{HCl}$ or $\mathrm{NaOH}$ were added to the tank, fresh glycerol was used for each experiment in order to avoid a significant decrease of viscosity. The stirred vessel was fully illuminated and a digital camera was placed in a tripod in front of the tank for capturing images of the mixing evolution.

In regard to the mixing times measurement in stirred vessels, an image processing method was developed using Microsoft Visual $\mathrm{C}++$ as programming language and an open computer vision library (Open CV) running in a Pentium IV PC computer, under Windows XP. The use of this method reduced the subjectivity of the estimation of mixing times by the human eye. First, a $7 \times 7$ median filter was applied to even background variations in order to enhance the images. Then, a segmentation process was developed obtaining the regions of interest by using the Hue $(H)$ and Light $(L)$ from the HLS color model [22]. The hue is expressed as an angle between $0^{\circ}$ and $360^{\circ}$ beginning with red at $0^{\circ}$, green at $120^{\circ}$ and blue at 
$240^{\circ}$, yellow, cyan and magenta are located at $60^{\circ}$, $180^{\circ}$ and $300^{\circ}$, respectively. On the other hand, light is a value that described the color intensity into the range of $[0,1]$.

The evolution of the mixing process in our experimental conditions is noticed by the changes from dark blue to dark orange as shown Figure 2. In this sense, the dark orange color showed the mixed $(M)$ regions, while some other different color showed the unmixed $(U M)$ regions [11]; mixing classes $(M, U M)$ were determined by evaluating $H$ and $L$ in a set of images, where $M$ corresponded to pixels with $H$ values ranging from $0^{\circ}$ to 50 and $L>0.5$. On the other hand, UM regions corresponded to pixels with $H$ values greater than $50^{\circ}$. In order to segment only areas of interest, the impeller, the agitation shaft and the region outside the tanks were removed. It is important to point out that the methodology was tested in a set of trained images having a good agreement in general terms in comparison with the mixing level obtained by reference. In this sense, the reference was made by a trained observer who manually made the segmentation and classification of mixing regions in the training set images. The largest error value reached with this method is $5 \%$, compared with the reference.

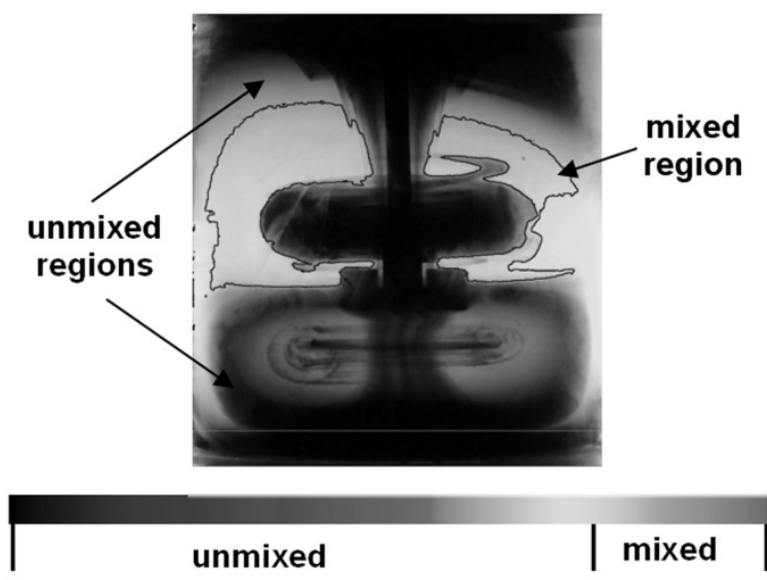

Figure 2. Color range for the classification of the mixed process.

\subsection{Velocity fields}

The flow patterns inside the stirred vessels were visualized by means of the particle image velocity (PIV) technique, using a commercial system (Dantec Dynamics, Inc.). A pulsed Nd:YAG laser having energy of $120 \mathrm{~mJ}$ and a wavelength of 532 $\mathrm{nm}$ was used as illumination source. For that purpose, an optical array was used to create a light sheet of less than $1 \mathrm{~mm}$ width so that the stirred vessel was illuminated in a specific plane. A CCD camera was placed perpendicularly to the illuminated plane. The camera was coupled and synchronized with the laser for capturing 15 pairs of images per second of the illuminated plane. Hollow spheres silver covered with a diameter of $10 \mu \mathrm{m}$ were used as particle tracers. The images were recorded once the hydrodynamic steady conditions were reached and the flow structures generated by the impellers remained stable. Mixing experiments were performed at room temperature $\left(\sim 24^{\circ} \mathrm{C}\right)$.

\section{Results}

\subsection{Mixing times}

An example of the mixing evolution using a single Rushton turbine centered and rotating steadily in one direction is shown in Table 1.

The homogeneity level in the first column is the result of applying the HLS based image analysis method. As mentioned earlier, neither the agitation shaft nor the impellers were taken into account for the analysis as areas of interest.

Figure 3 plots the level of homogeneity as function of time for the different scenarios investigated. As expected, the lowest homogeneity level is in general obtained under steady stirring conditions with the single Rushton turbine centered. Since the Rushton turbine discharges flow in the radial direction, a very limited pumping capacity is obtained resulting in null homogenization during the first 40 minutes. As the impeller is displaced from the vessel centerline, the fluid is homogenized; however, no improvement is noticed after 60 minutes with respect to the centered case. 


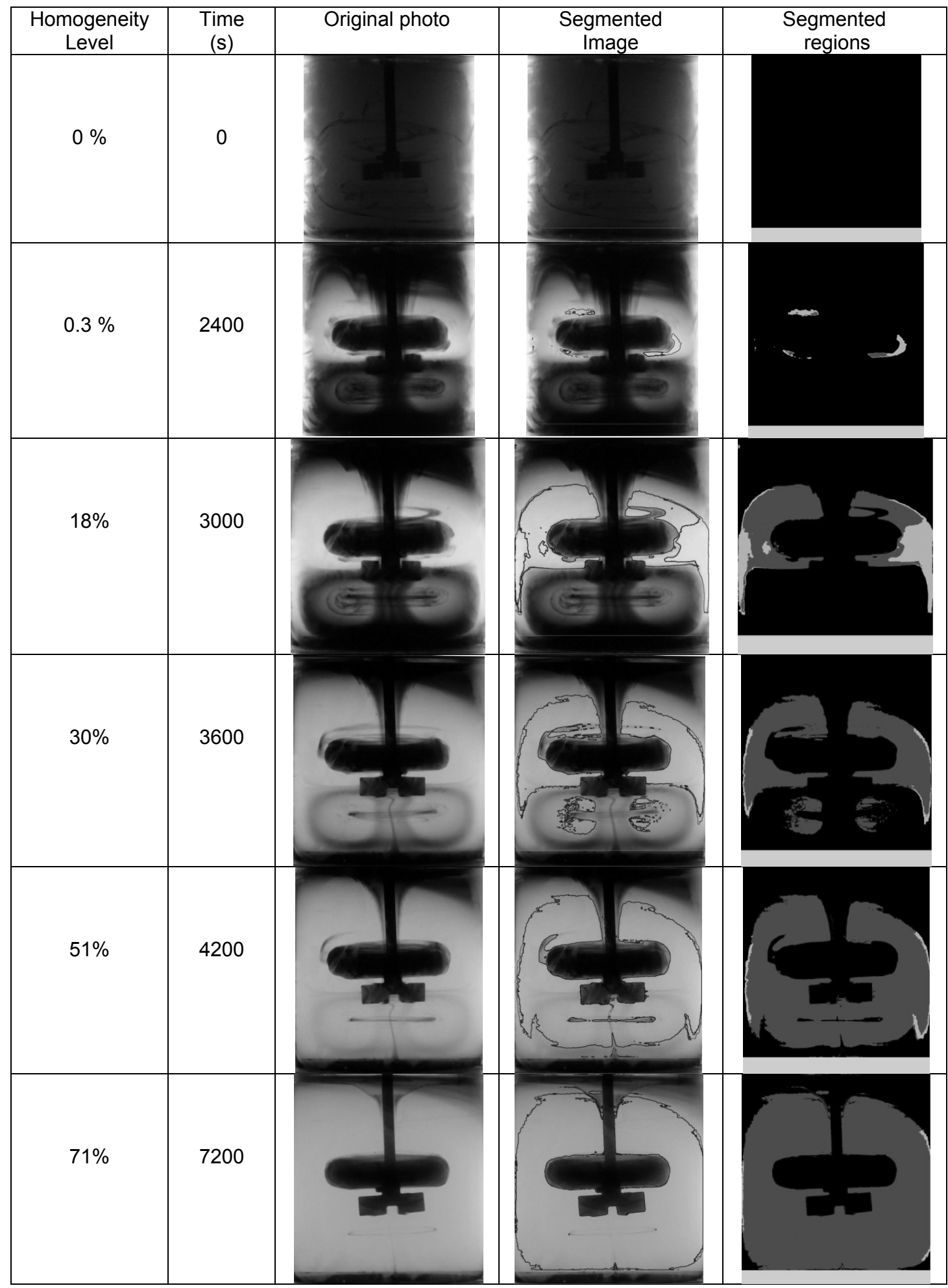

Table 1. Mixing evolution with a centered Rushton turbine. 


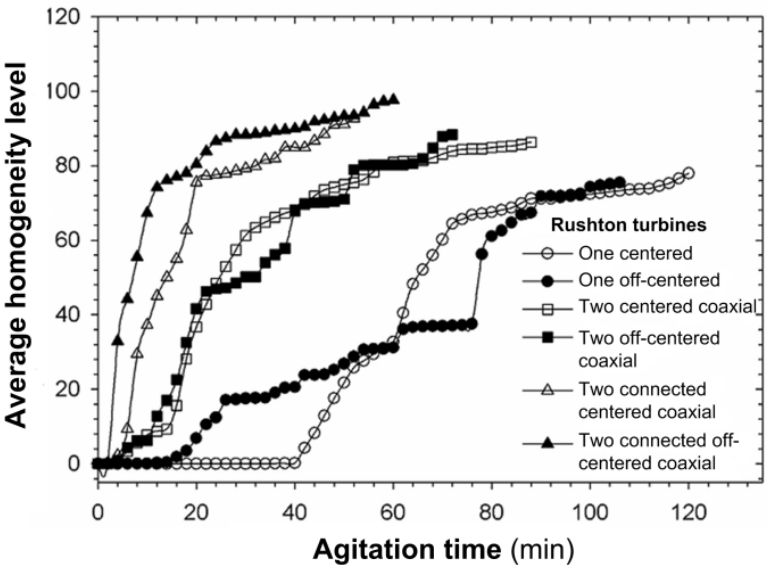

Figure 3. Homogeneity level as a function of time.

In general, the use of two coaxial Rushton turbines irrespective of the radial position with respect to the vessel centerline gives better results. After 32 minutes a homogenization level of $48 \%$ and $64 \%$ is obtained with the two impellers array centered and off-centered, respectively. After 40 minutes, the trends for both scenarios are quite similar.

Finally, the best results are obtained when using two coaxial impellers connected by the thin plates. After 15 minutes, a homogeneity level of about $52 \%$ and $76 \%$ is obtained with this array in the central and off-centered position, respectively. Since connecting plates result in a larger impeller surface, the pumping capacity is enhanced; however, higher energy consumption is required to achieve the desired homogeneity level.

\subsection{Energy consumption}

Figure 4 plots the energy consumption as a function of the homogeneity level. In the present case, the energy is determined from the following expression:

$$
E=2 \pi N \int_{0}^{t} \tau d t
$$

where $N$ is the rotational speed of the impeller (rev/s), $\tau$ is the torque (N.m) and $t$ is the agitation time (s) for a specific homogeneity level.

As Figure 4 shows, lower energy is required when the flow into the vessel is continuously perturbed by off-centering the impellers. This is in general true, except for the case of the single turbine. More mixing energy is required to achieve a level between $28 \%$ and $64 \%$ if the turbine is placed offcentered. On the other hand, if the single impeller configuration is compared with the other arrays, about $36 \%$ and $73 \%$ less energy is needed when using the two coaxial impellers without and with the connecting plates, respectively, for a homogeneity level of $64 \%$. Particularly, the best results are obtained with the two coaxial impellers connected by the thin plates.

Hence, the use of the two coaxial impellers with the connecting plates is an attractive alternative to achieve a good homogeneity level in shorter time while requiring lower energy consumption. The connecting plates promote a stronger flow from the tank bottom to the surface resulting in an improved pumping capacity. This will be shown in a quantitative manner in the next section.

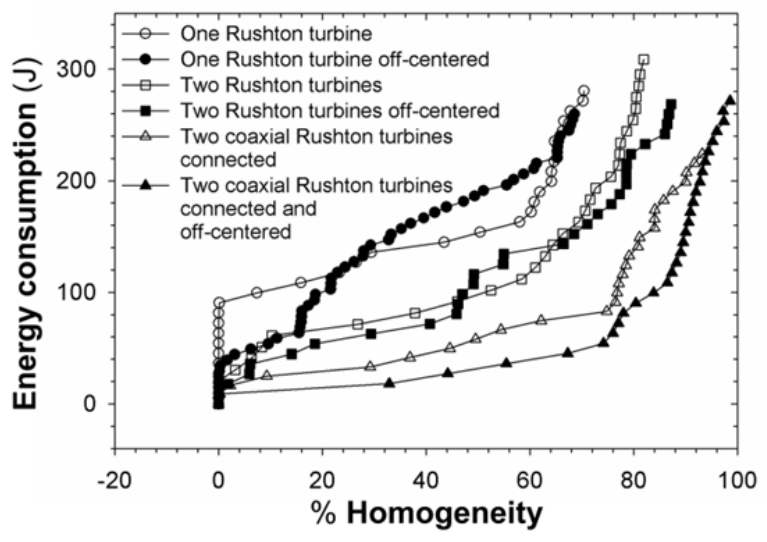

Figure 4. Energy consumption as a function of homogeneity level.

\subsection{Velocity fields}

To understand the cause of the improvement in the mixing rate with the new impeller geometry, a PIV study was conducted to analyze the velocity field in the tank. The secondary flows in the mixer were analyzed for a range of conditions. Figure 5 shows a typical flow field obtained with the PIV technique; it shows the velocity field in an r-z plane. In it, the appearance of secondary flows is very clear. The pumping action of the impeller is characterized by an axial flow on top and bottom of the impeller; the fluid is then expelled radially at the height of the impeller. 


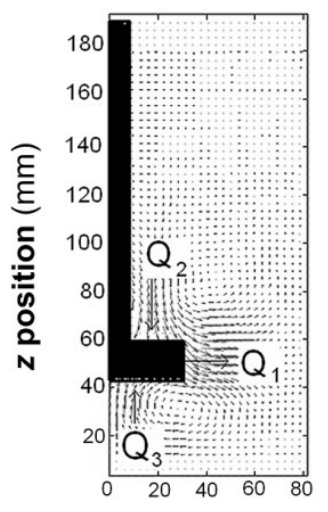

$r$ position $(\mathrm{mm})$

Figure 5. Typical velocity fields around a Rushton turbine.

In order to verify the validity of the PIV measurements, a mass conservation balance of the flow through the impeller was conducted. From Figure 5 , the incoming flow $\left(Q_{2}\right.$ and $\left.Q_{3}\right)$ should be equal to the outgoing flow $\left(Q_{1}\right)$ :

$$
Q_{1}=Q_{2}+Q_{3}
$$

These volumetric flows can be calculated from the PIV measurements considering

$$
Q=\int V d A
$$

where $V$ is the fluid velocity passing through an area $A$. For each of these volumetric flows, the area is that occupied by the top circle and the bottom circle of the impeller $\left(Q_{2}+Q_{3}\right)$ or the area radially projected by the six blades of the impeller $\left(Q_{1}\right)$. The calculations of $Q_{1}, Q_{2}$ and $Q_{3}$ for all scenarios investigated are reported in Table 2.

From these measurements, it is possible to understand the differences in the performance of the different configurations. Clearly, the magnitude of the induced flows increases with impeller effective area. In turn, the mixing time is reduced for larger induced flows. The improvement in the mixing process is, therefore, achieved by an increase of the impeller area and the strength of induced secondary flows within the tank. The twocoaxial Rushton turbines connected by two thin plates, increases the strength of the secondary flows by a factor of 2.2 with respect to the single Rushton turbine case; however, the energy consumption only increases by a factor of 2 . The increase in the energy consumption is therefore compensated positively by a reduction in mixing time.

\begin{tabular}{|c|c|c|c|c|}
\hline Scenario & $\begin{array}{c}\text { Incoming } \\
\text { flow } \\
\left(Q_{2}+Q_{3}\right) \\
\mathrm{m}^{3} / \mathrm{s} \\
(1 \mathrm{e}-6)\end{array}$ & $\begin{array}{c}\text { Outgoin } \\
\text { g flow } \\
\left(Q_{1}\right) \\
\mathrm{m}^{3} / \mathrm{s} \\
(1 \mathrm{e}-6)\end{array}$ & $\begin{array}{c}\text { Difference } \\
\mathrm{m}^{3} / \mathrm{s} \\
(1 \mathrm{e}-8)\end{array}$ & $\begin{array}{c}\% \\
\text { Error }\end{array}$ \\
\hline $\begin{array}{c}1 \mathrm{RT} \\
\text { centered }\end{array}$ & 1.160 & 1.134 & 2.6 & 2.25 \\
\hline $\begin{array}{c}1 \mathrm{RT} \text { off- } \\
\text { centered }\end{array}$ & 1.097 & 1.114 & 1.7 & 1.53 \\
\hline $\begin{array}{c}2 \mathrm{RT} \\
\text { centered }\end{array}$ & 1.476 & 1.510 & 4.4 & 2.25 \\
\hline $\begin{array}{c}2 \mathrm{RT} \text { off- } \\
\text { centered }\end{array}$ & 1.448 & 1471 & 2.3 & 1.56 \\
\hline $\begin{array}{c}2 \mathrm{RT} \\
\text { connected } \\
\text { centered }\end{array}$ & 2.391 & 2.377 & 1.4 & 0.59 \\
\hline $\begin{array}{c}2 \mathrm{RT} \\
\text { connected } \\
\text { off- } \\
\text { centered }\end{array}$ & 2.545 & 2.482 & 6.3 & 2.54 \\
\hline
\end{tabular}

Table 2. Flow rate for the different impeller configurations.

Note also that, in general, the magnitude of the induced flow is reduced for the off-center configurations. The only arrangement in which an improvement is observed is that of the two Rushton turbines connected by two thin plates. For this particular case, an increase of $6 \%$ of the strength of the induced flow, with respect to the centered impeller with the same geometry, for practically the same power consumption.

\subsection{Flow patterns for different impeller configurations}

In addition to the quantitative measurements obtained with the PIV system, it also possible to visualize the flow structures within the measuring plane. In this manner, it is possible to further understand the differences in performance observed by the different impeller configurations. Hence, we obtained contour plots and stream lines of the induced flows. Furthermore, in this investigation visualizations of the flow in planes to the left and right of the impeller are obtained. For the case of centered impellers, this is not necessary due to the axial symmetry of the flow; hence, for these cases only an half of the tank is shown. For the off-centered cases, however, the 
differences between the flows 'near' and 'far' from the wall may be important. In this case, the image of the whole tank is shown. Note that these visualizations (left and right) were not taken simultaneously.

Figures 6 and 7 show the flow visualizations for the single and dual Rushton turbine impellers, both for the centered and off-centered cases.

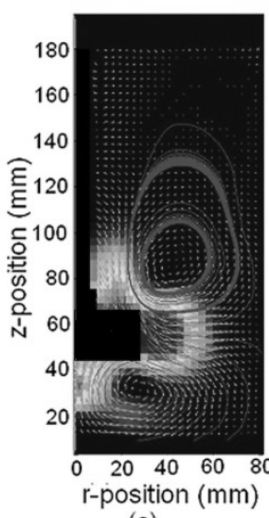

(a)

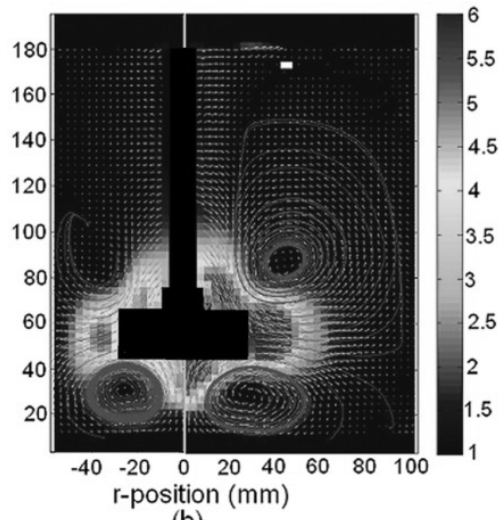

(b)
Figure 6 . Velocity fields generated by one Rushton turbine, a) centered and b) off-centered (bar scale in $\mathrm{mm} / \mathrm{s}$ ).

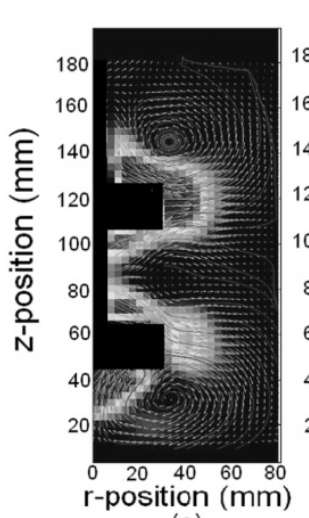

(a)

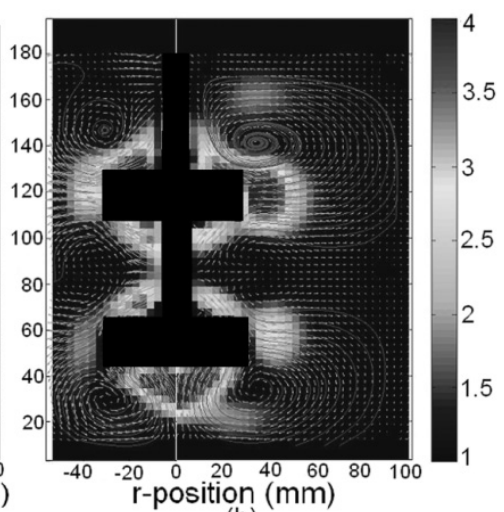

(b)
Figure 7 Velocity fields generated by two Rushton turbines, a) centered and b) off-centered (bar scale in $\mathrm{mm} / \mathrm{s}$ ).

The images show the velocity fields (vectors), the fluid velocity magnitude (colors) and the stream line patterns (lines). The stream lines were generated from points near the tip of the blades. In this manner, the vortical structures were clearly visualized.

It is observed that a vortex structure is readily formed around the impeller in all cases. The vortices were stable and appear as a result of radial flow induced by the impellers. The discharge induces a pressure around the center of the impeller which is occupied by the fluid on the top and bottom part of the impeller. This cycle is repeated continuously. For the case of the two coaxial Rushton turbines connected by thin plates, images were taken at three different angular positions. As Figure 8 shows, the flow pattern for this configuration changes for the different angular positions around the impeller. The flow patterns shown in Figure 8 were obtained for different blade angular positions $\left(0^{\circ}, 60^{\circ}\right.$ and $\left.120^{\circ}\right)$ being the blade tip the reference.

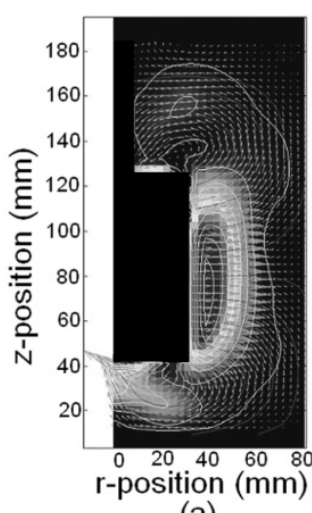

(a)

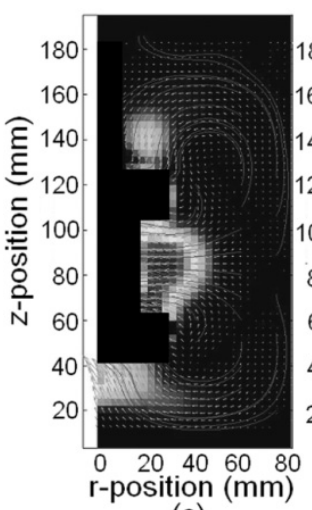

(c)

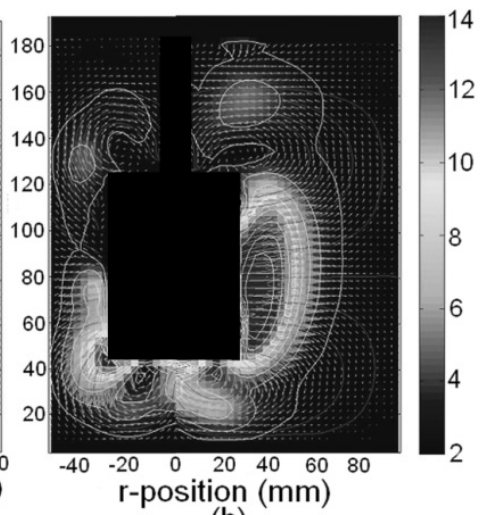

(b)

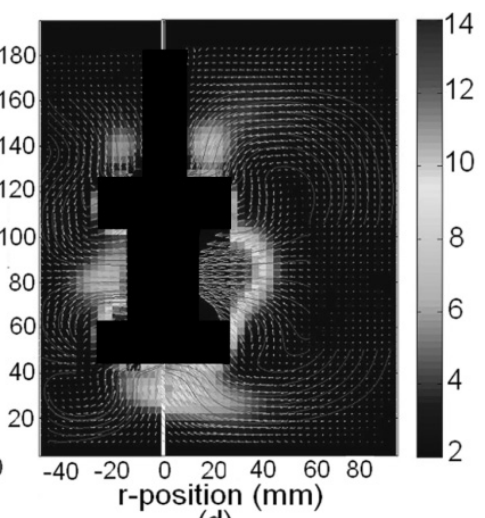

(d) 


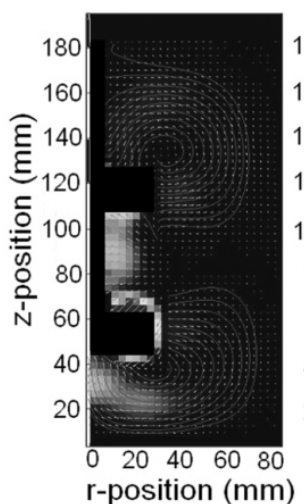

(e)

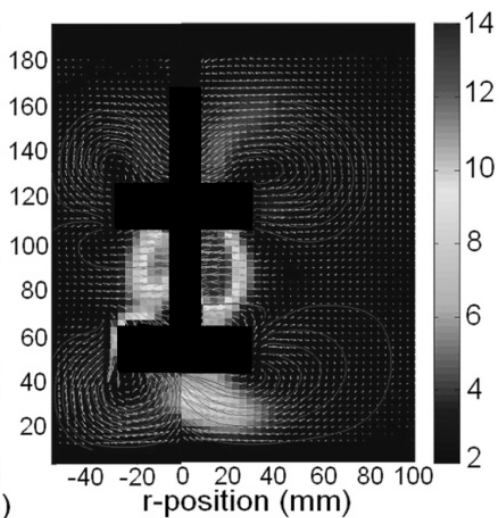

(f)
Figure 8 . Velocity fields generated by two Rushton turbines coupled by two thin plates spaced at: a) $0^{\circ}$ centered; b) $0^{\circ}$ off-centered; c) $60^{\circ}$ centered; d) $60^{\circ}$ offcentered; e) $120^{\circ}$ centered; f) $120^{\circ}$ off-centered.

Clearly, for this configuration, the plates that connect the two turbines have a significant effect in the flow pattern. The plates improve the circulation and enhance the mixing achieved in the tank. The measured values of the velocity magnitude can reach values up to two times those measured in the other, simpler, configurations. This fact can be clearly observed in Figures 8(a) and 8(b). The enhanced fluid motion inside the mixing tank can be explained by the fact that when the plates pass around the tank, while rotating, the direction of the radial flow changes completely. Ahead of the plates, the flow is pushed radially outwards; behind them, the fluid is dragged radially inwards. This periodic change of direction in the flow pattern is responsible for the increase mean velocity and mixing capacity of this device. Furthermore, it is clearly observed that the offcentered configuration produces larger values of the velocity inside the tank. Since the wall proximity is different for opposite blades, larger fluxes can be induced for the case of the blades which are further away from the wall. The estimated error for all PIVsystem experiments is $3 \%$ maximum, which was obtained by matching the number of pixels and their relationship to $\mathrm{mm}$.

\subsection{Vortex structure}

The velocity fields obtained from the PIV technique can be used to calculate the vorticity of the flow.
Mathematically, the vorticity is defined as the curl of the velocity field

$$
\varpi=\nabla \times \bar{v}
$$

For the 2D flow fields experimentally obtained, it is possible to obtain the vorticity component perpendicular to the plane of measurement. Considering a $(r, z)$ coordinate system, the vorticity is defined as

$$
\omega=\frac{\partial U_{z}}{\partial r}-\frac{\partial U_{r}}{\partial z}
$$

Positive and negative values correspond to vortex rotating in counter-clockwise and clockwise direction, respectively.

Figures 9 and 10 show the vorticity maps obtained for the single and dual Rushton turbine arrangements, in the centered and off-centered configurations. In both figures, the vorticity fields revealed a spiral motion with tendency to swirl around a center in the azimuthal direction, i.e., coherent vortices can be identified. Moreover, the zones of highest vorticity are located in the vicinity of the impellers, which can explain the formation of the doughnut-shaped structures on the flow above and below the impeller. Far from the impeller, the vorticity has small values, which coincide with zones in which poor mixing (long mixing times) is detected (as seen previously in Section 3.1). Note that the values of vorticity are, in fact, larger for the case of the single Rushton turbine. We can explain this fact from the pressure gradient caused by the impellers. For a given set of conditions, the same pressure gradient is induced in the mixing tank. For the dual impeller, the gradient is caused by the action of the two impellers, as opposed to the single impeller, in which all the motion is induced by one turbine. Hence, we can argue that the high vorticity regions have a larger strength for the single impeller case. However, the vorticity field induced by the dual impeller case is extended over a larger area, which leads to an improvement of the mixing rates. 


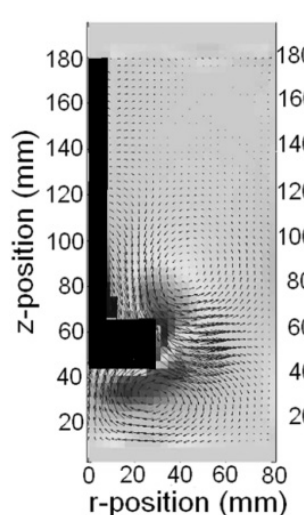

(a)

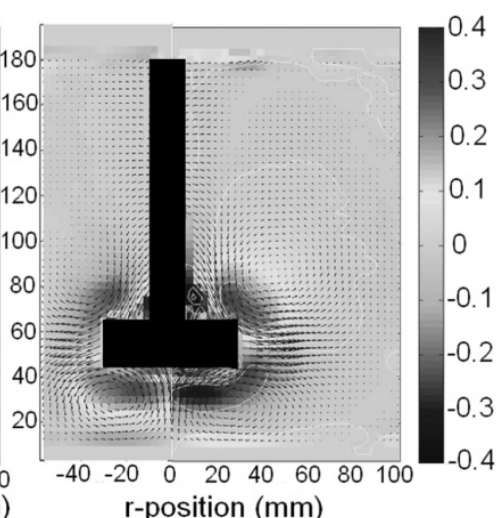

(b)
Figure 9. Vorticity fields velocity generated by one Rushton turbine a) centered and (b) off centered(bar scale in $\mathrm{s}^{-1}$ ).

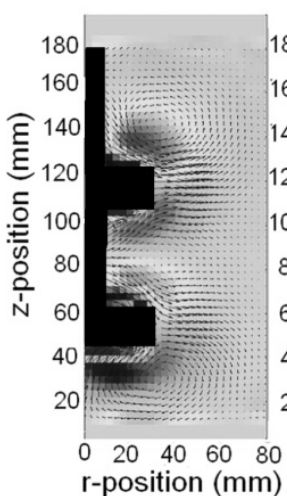

(a)

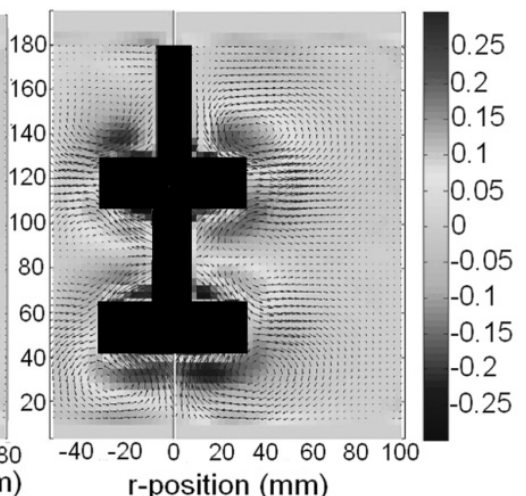

(b)
Figure 10. Vorticity fields velocity generated by two Rushton turbines, (a) centered and

(b) off-centered (bar scale in $\mathrm{s}^{-1}$ ).

Figure 11 shows the vorticity fields obtained for the dual impeller with plates for different angular positions. As before, since the axi-symmetry is lost in this case, a series of measurements following the procedure described in Section 2.4 were made for three different angular positions. The vortical structures are largely elongated as the impeller rotates. Large differences in the vorticity field are clearly observed for the three angular positions. We can attribute the observed enhancement of the mixing performance to the fact that the vortical structures are being continuously modified; the expansion and contraction of these structures induce fluid agitation which, in turn, enhances the mixing in the tank.
As in the previous cases, the off-centered configuration induces gradients of vorticity in the radial direction. For the case of the mixer with two turbines connected with plates, gradients of vorticity are also produced in the angular direction. The combined variation of vorticity in angular and radial directions induces a better mixing performance for this case.

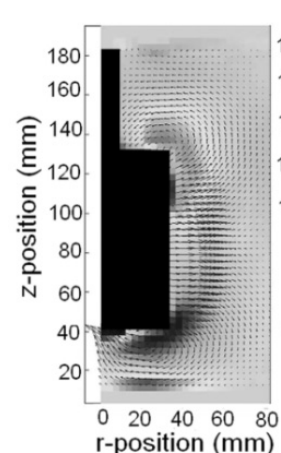

(a)

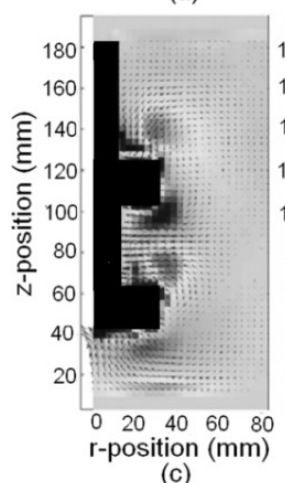

(c)

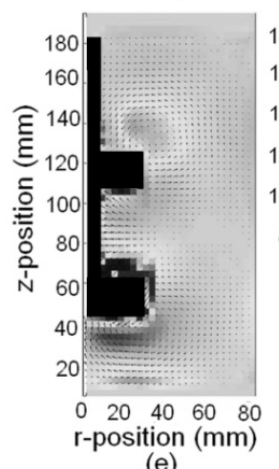

(e)

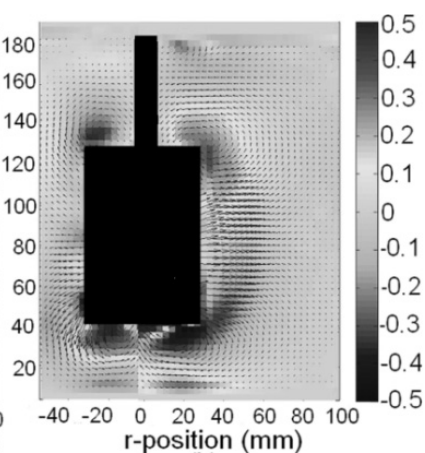

(b)

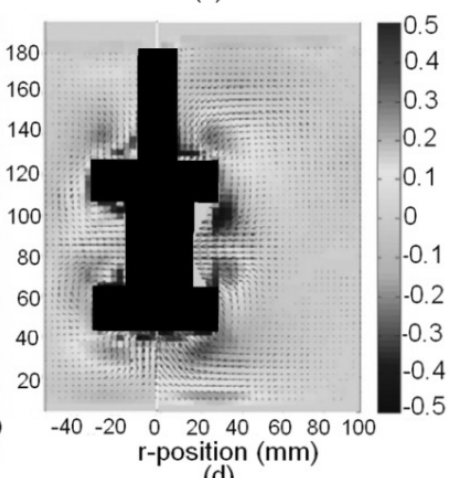

(d)

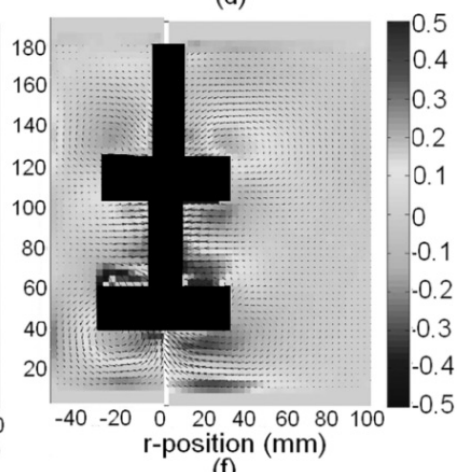

(f)

Figure 11. Vorticity fields generated by two Rushton turbines coupled by two thin plates spaced at $180^{\circ}:$ a) position 0 degrees centered; b) position 0 degrees offcentered; c) position 60 degrees centered; d) position 60 degrees off-centered; e) position 120 degrees centered; f) position 120 degrees off-centered (bar scale in $\mathrm{s}^{-1}$ ). 


\subsection{Vertical velocity $\left(U_{z}\right)$ profiles}

In addition to the visualization of the flow fields in the tank, quantitative information can also be obtained from the PIV data. The vertical velocity $\left(U_{z}\right)$ was evaluated to determine the differences among the different impeller configurations studied in this work. This analysis was carried out considering a normalized vertical velocity of the fluid, defined as

$$
V^{*}=\frac{V_{\text {fluid }}}{V_{\text {impeller }}}
$$

where $V_{\text {fluid }}$ is the vertical velocity $\left(U_{z}\right)$ of the fluid in a given position, and $V_{\text {impeller }}$ is the impeller tip speed. The position of the measuring point is normalized by

$$
R^{*}=\frac{r_{\text {fluid }}}{r}
$$

where $r_{\text {fluid }}$ is the distance of the measuring point from the center of the tank and $r$ is the impeller radius. The height of the measuring point is normalized by

$$
H^{*}=\frac{H_{\text {fluid }}}{H}
$$

where $H_{\text {fluid }}$ is the height of measuring point and $H$ is the level liquid in the tank. The normalized velocity is plotted as a function of normalized position in Figures 12 to 14 . These plots show the velocity profiles considering different values of the axial position, for all the impellers tested in this study.

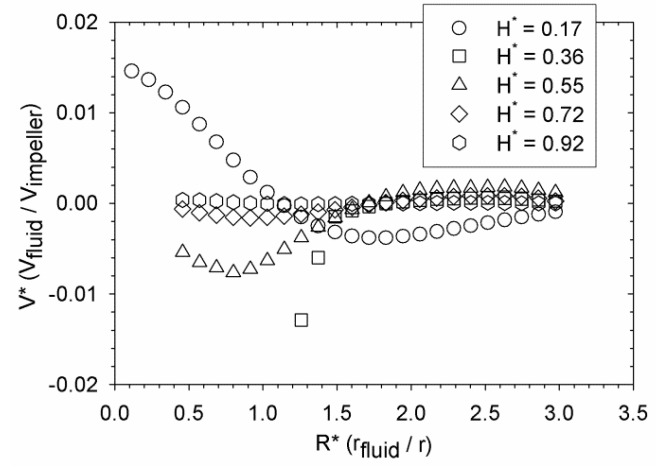

(a)

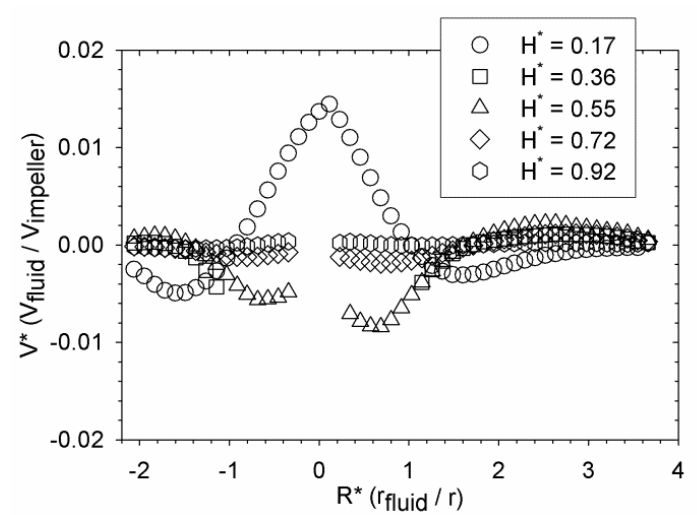

(b)

Figure 12. Vertical velocity by one Rushton turbine: (a) centered; (b) off-centered.

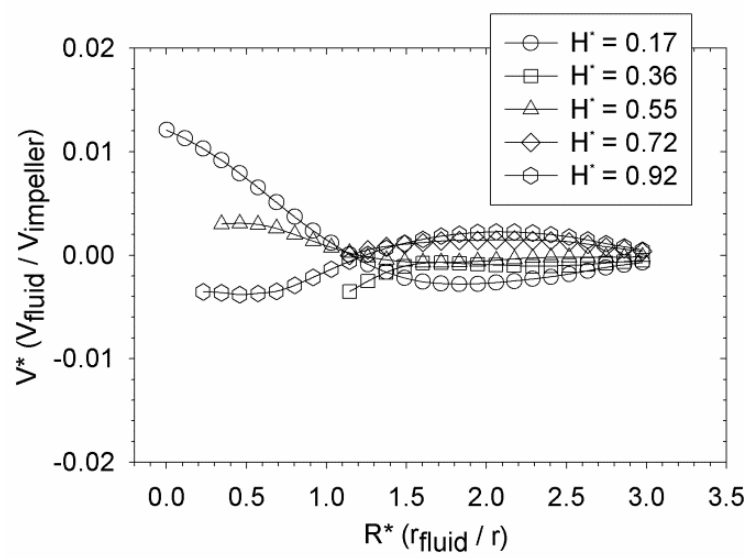

(a)

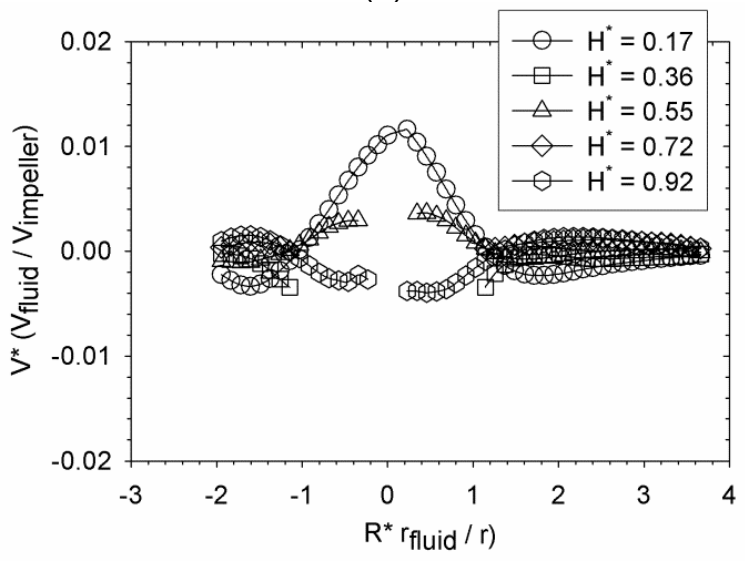

(b)

Figure 13. Vertical velocity by two Rushton turbines: (a) centered; (b) off- centered. 
The shape of the velocity profiles changes depending on the existing vertical circulation induced by each impeller configuration. These results suggest that the dual turbine impeller with the two connecting plates induces larger axial velocity components. In Figures. 12 and 13 the profiles $\left(U_{z}\right)$ show similar tendencies which correspond to the single and dual Ruston turbine configurations. For the case of the impeller with the connecting plates (see Figure 14), the tendency is modified as much larger values of the induced vertical motion are observed. A low pressure region appears in the region behind these plates which, in turn, induce a pressure gradient with the rest of the tank. This gradient is responsible for the additional fluid motion observed for this impeller. For the case of the off-centered impeller, the mixing efficiency is improved because the induced vertical motion occurs in a larger area on the side far from the wall.

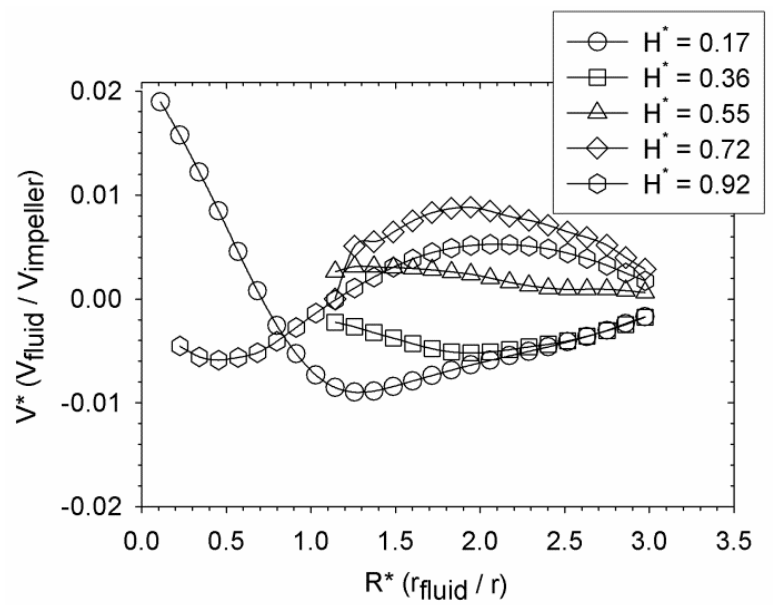

(a)

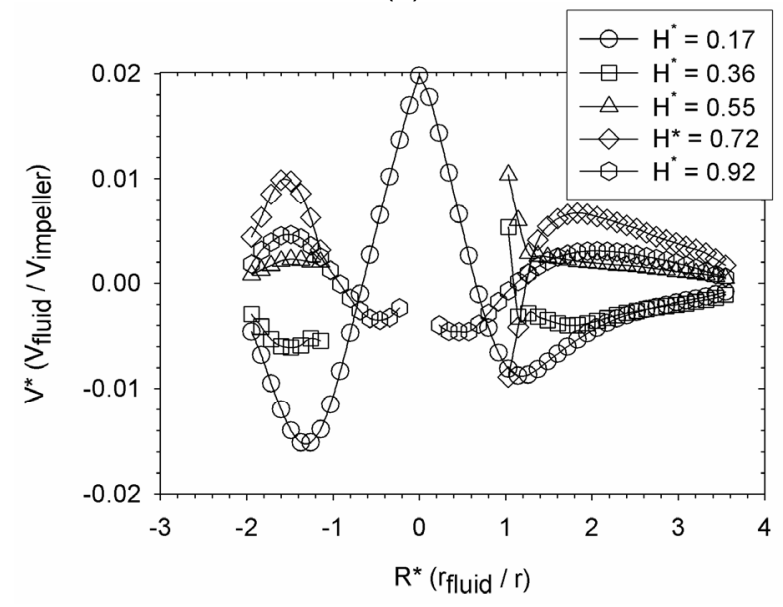

(b)

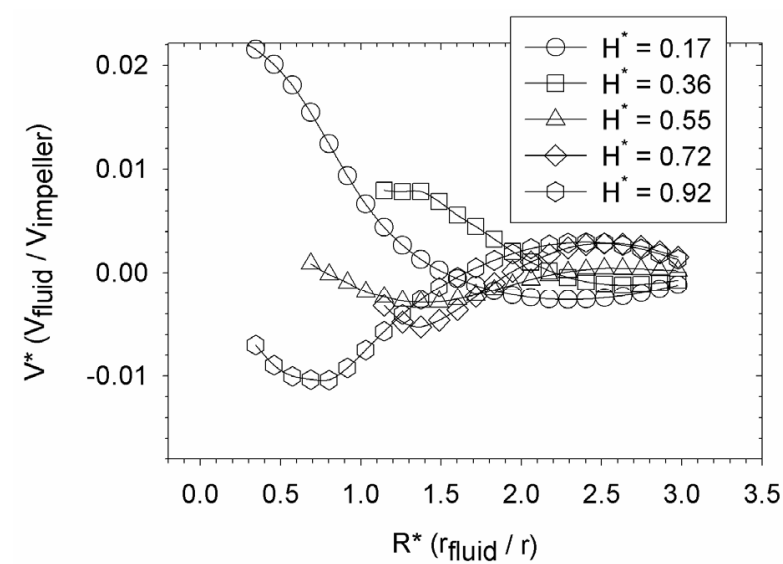

(c)

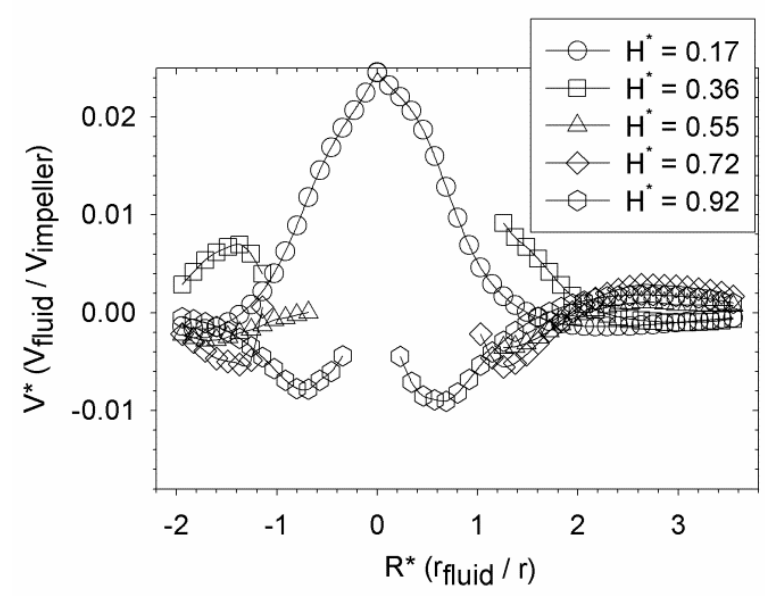

(d)

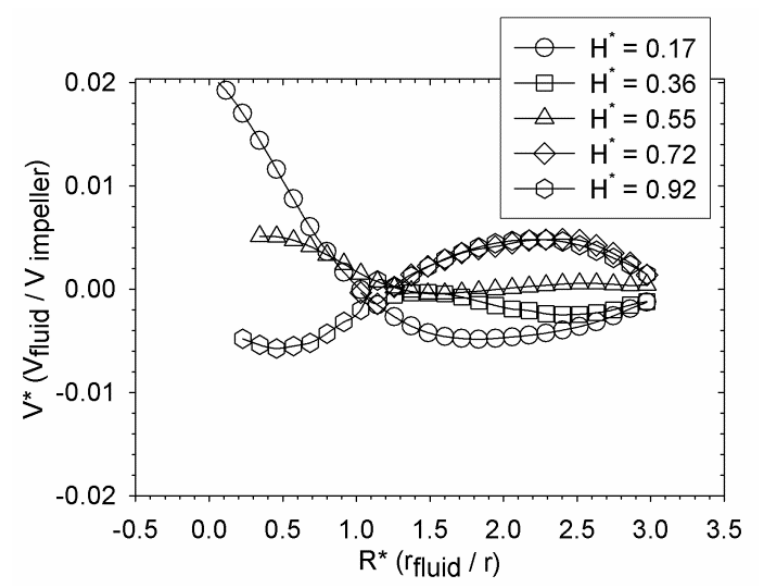

(e) 


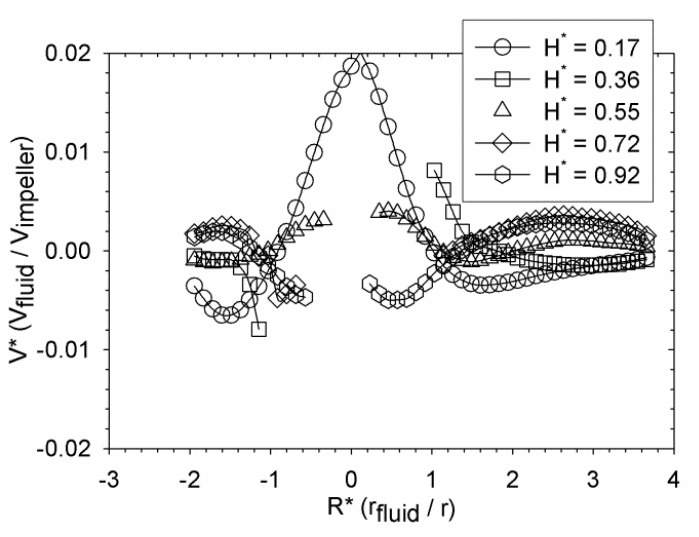

(f)

Figure 14. Vertical velocity generated by two Rushton turbines coupled by two thin plates spaced at $180^{\circ}:(a)$ position 0 degrees centered; (b) position 0 degrees offcentered; (c) position 60 degrees centered; (d) position

60 degrees off-centered; (e) position 120 degrees centered; (f) position 120 degrees off-centered.

\subsection{Pumping capacity}

The pumping capacity has been determined for the different impeller configurations in terms of the pumping number, which is defined by the following equation:

$$
N q(z)=\frac{Q_{z}}{N D^{3}}
$$

where $N$ is the rotational speed (rev/s), $D$ is the impeller diameter $(\mathrm{m})$ and $Q_{z}$ is the volumetric flow expelled by the turbine, defined by

$$
Q_{z}=\int_{0}^{r} 2 \pi r\left\langle V_{z}\right\rangle d r
$$

$Q_{z}$ is the volumetric flow rate in the Z-axis direction of fluid crossing through an area around the impeller, at a distance $1.63 \mathrm{~mm}$ from the tip impeller blade. $\left\langle V_{z}\right\rangle$ is the mean vertical velocity obtained by using the PIV technique previously described. This velocity is the average value of 3000 individual measurements taken at intervals of $480 \mathrm{~ms}$, which statistically ensures the reliability of the measurements. In the case of the off-centered scenarios, $Q_{z}$ was the sum of the flow rate in the region close to the vessel wall and the flow rate in the region far from the vessel wall. Table 3 summarizes the pumping number in the vertical direction obtained for the scenarios investigated. Similar results in the pumping capacity were found by Rice et al.[23] for one Rushton turbine centered at very low $R e$ number.

\begin{tabular}{|c|c|c|}
\hline \multirow{2}{*}{ Impellers } & Position & $N q(\mathrm{z})$ \\
\hline \multirow{2}{*}{ 1 RT } & Centered & 0.11 \\
\cline { 2 - 3 } & Off-centered & 0.21 \\
\hline \multirow{2}{*}{$2 \mathrm{RT}$} & Centered & 0.28 \\
\cline { 2 - 3 } & Off-centered & 0.29 \\
\hline \multirow{2}{*}{$\begin{array}{c}\text { 2 RT } \\
\text { connected }\end{array}$} & Centered & 0.35 \\
\cline { 2 - 3 } & Off-centered & 0.36 \\
\hline
\end{tabular}

Table 3. Pumping number for the different scenarios.

The benefits of using off-centered impellers under the laminar regime have been well recognized and reported elsewhere $[7,11,12,13]$. In the case of a single Rushton turbine, the pumping number under off-centered conditions is almost twice than the one obtained with the impeller centered. However, the effect of eccentricity is lower when using two coaxial impellers especially in the case of the 2 Rushton turbines connected by the thin plates.

\section{Conclusions}

Two different techniques, colorimetry and particle image velocimetry, have been used to investigate the effect of eccentricity and some impeller configurations on unbaffled tanks in the laminar regime with a viscous Newtonian fluid. It has been demonstrated that shorter mixing times, lower energy draw and better pumping capacity are in general obtained when using off-centered impellers. The best results have been obtained with single impellers slightly displaced from the vessel centerline, which is in good agreement with the findings reported in the literature. Although, less energy is required with off-centered impellers to achieve a certain degree of homogenization in the stirred vessel, less benefit in the pumping capacity is obtained with two coaxial impeller configurations in the off-centered conditions. In general terms, a novel configuration consisting of a dual turbine with connecting plates exhibits better mixing performance by reducing the mixing time and improves the homogeneity level in a batch 
system, even if higher energy is required to achieve a certain degree of homogeneity.

\section{Acknowledgements}

The financial support from DGAPA-UNAM grant IN-108312 and from National Council of Science and Technology (CONACyT) grant 123840 are highly appreciated. The technical advice and assistance during PIV experiments from Carlos Palacios is also acknowledged.

\section{References}

[1] L. Bresler, T. Shinbrot, G. Metcalfe, J.M. Ottino, "Isolated mixing regions: origin, robustness and control", Chem. Eng. Sci., vol. 52, no. 10, pp. 1623-1636, 1997.

[2] P. Mavros, "Flow visualization in stirred vessels, A review of experimental techniques", Trans. Inst. Chem. Eng. Part A, vol. 79, no.2, pp. 113-127, 2001.

[3] A.B. Norwood, A.B. Metzner, "Flow patterns and mixing rates in agitated vessels", AIChE J., vol. 6, no. 3, pp. 432-437, 1960.

[4] T.P. Elson, D.J. Cheesman, A.W. Nienow, "X-ray studies of cavern sizes and mixing performance with fluids possessing a yield stress", Chem. Eng. Sci., vol. 41 , no. 10 , pp. 2555-2562, 1986.

[5] P.J. Holden, M. Wang, R. Mann, F.J. Dickin, R.B. Edwards, "On detecting mixing pathologies inside a stirred vessel using electrical resistance tomography", Trans. Inst. Chem. Eng. vol. 77, no. 8, pp. 709-712, 1999.

[6] E. Brito-de la Fuente, S. Hernández, A. Segura, L. Medina, P.A. Tanguy, G. Ascanio, "Laminar mixing in stirred tanks using dynamics perturbations and nonsymmetric geometry conditions", Proceedings of the VI Pan-American Congress in Applied Mechanics, Rio de Janeiro, Brazil, 1999.

[7] G. Ascanio, M. Brito-Bazan, E. Brito-de la Fuente, P.J. Carreau, P.A. Tanguy, "Unconventional configuration studies to improve mixing times in stirred tanks", Can. J. Chem. Eng. vol. 80, no. 4, pp. 558-565, 2002.

[8] H. Aref, "Stirring by chaotic advection", J. Fluid Mech., vol. 143, pp. 1-21, 1984.

[9] H. Aref, S. Balachandar, "Chaotic advection in a Stokes flow", Phys. Fluids. vol. 29, no. 11, 1986, pp. 3515-3521, 1986.

[10] J.G. Franjione, C.W. Leong, J.M. Ottino, "Symmetries within chaos: A route to effective mixing". Phys. Fluids, vol. 1, no. 11, pp. 1772-1783, 1989.
[11] M. Alvarez, P.E. Arratia, F.J. Muzzio, "Laminar mixing in eccentric stirred tank systems", Can. J. Chem. Eng., vol. 80, no. 4, pp. 546-557, 2002.

[12] G. Montante, A. Bakker, A. Paglianti, F. Magelli, "Effect of the shaft eccentricity on the hydrodynamics of unbaffled stirred tanks", Chem. Eng. Sci., vol. 61, no. 9, pp. 2807-2814, 2006.

[13] J. Karcz, M. Cudak, J. Szoplik, "Stirring of a liquid in a stirred tank with an eccentrically located impeller", Chem. Eng. Sci., vol. 60, no. 8-9, pp. 2369-2380, 2005.

[14] C. Galletti, E. Brunazzi, "On the main flow features and instabilities in an unbaffled vessel agitated with an eccentrically located impeller", Chem. Eng. Sci., 63(18), 4494-4505, 2008.

[15] C. Galletti, S. Pintus, E. Brunazzi, E. "Effect of shaft eccentricity and impeller blade thickness on the vortices features in an unbaffled vessel", Chem. Eng. Res. Des., vol. 87, no. 4, pp. 391-400, 2009.

[16] J.M. Ottino, C.W. Leong, H. Rising, P.D. Swanson, "Morphological structures produced by mixing in chaotic flows", Nature, vol. 333, pp. 419-425, 1988.

[17] P.D. Swanson, J.M. Ottino, "Comparative computational and experimental study of chaotic mixing of viscous fluids", J. Fluid Mech. vol. 213, 1990, pp. 227-249.

[18] F.J. Muzzio, P.D. Swanson, J.M. Ottino, The statics of stretching and starting in chaotic flows, Phys. Fluids, vol. A 3, no. 5, pp. 822-834, 1991.

[19] F.J. Muzzio, C. Meneveau C., P.D. Swanson, J.M. Ottino, "Scaling and multifractal properties of mixing in chaotic flows", Phys. Fluids, vol. A 4, no. 7, pp. 14391456, 1992.

[20] G. Ascanio, P.A. Tanguy, "Mixing of shear-thinning fluids with dual off-centered impellers", Can. J. Chem. Eng., vol. 83, no. 3, pp. 393-400, 2005.

[21] D.J. Lamberto, F.J. Muzzio, P.D. Swanson, "Using time-dependent RPM to enhance mixing in stirred vessels", Chem. Eng. Sci., vol. 51, no. 5, pp. 733-741, 1996.

[22] K. Plataniotis , A. Venetsanopoulos, "Color image processing and applications" Germany, Springer, 2000.

[23] M. Rice, J. Hall, G. Papadakis, M. Yianneskis, "Investigation of laminar flow in a stirred vessel at low Reynolds numbers", Chem. Eng. Sci., vol. 61, no. 9, pp. 2762-2770, 2006. 
